\title{
Using a trichromatic CCD camera for spectral skylight estimation
}

\author{
Miguel A. López-Álvarez,, ${ }^{1, \star}$ Javier Hernández-Andrés, ${ }^{2}$ Javier Romero, ${ }^{2}$ \\ F. J. Olmo, ${ }^{3}$ A. Cazorla, ${ }^{3}$ and L. Alados-Arboledas ${ }^{3}$ \\ ${ }^{1}$ Hewlett-Packard Spain, Large Format Printing Division, Sant Cugat del Vallès, Barcelona, Spain \\ ${ }^{2}$ Colour Imaging Laboratory, Departamento de Óptica, Universidad de Granada, 18071, Granada, Spain \\ ${ }^{3}$ Atmospheric Physics Group, Departamento de Física Aplicada, Universidad de Granada,18071, Granada, Spain \\ *Corresponding author: migangel@ugr.es
}

Received 28 April 2008; accepted 30 May 2008;

posted 27 June 2008 (Doc. ID 95569); published 16 July 2008

\begin{abstract}
In a previous work [J. Opt. Soc. Am. A 24, 942-956 (2007)] we showed how to design an optimum multispectral system aimed at spectral recovery of skylight. Since high-resolution multispectral images of skylight could be interesting for many scientific disciplines, here we also propose a nonoptimum but much cheaper and faster approach to achieve this goal by using a trichromatic RGB charge-coupled device (CCD) digital camera. The camera is attached to a fish-eye lens, hence permitting us to obtain a spectrum of every point of the skydome corresponding to each pixel of the image. In this work we show how to apply multispectral techniques to the sensors' responses of a common trichromatic camera in order to obtain skylight spectra from them. This spectral information is accurate enough to estimate experimental values of some climate parameters or to be used in algorithms for automatic cloud detection, among many other possible scientific applications. (c) 2008 Optical Society of America

OCIS codes: $\quad 150.2950,280.0280,040.0040$.
\end{abstract}

\section{Introduction}

When we enjoy the atmospheric optics phenomena related to the interaction between particles in the atmosphere and light—such as rainbows, halos, glories, or coronas-we are always interested in taking a picture of them with a simple digital charge-coupled device (CCD) camera. It would be very interesting to record at the same time the spectral information regarding this phenomenon in the same picture, but we do not often travel with a spectroradiometer since they are expensive, not portable, and provide only one spectrum per measurement. By doing this, for example, we could analyze the spectral characteristics of single pixels in a rainbow or a glory, hence making it possible to extend the comprehension of these beautiful and rare events. Multispectral systems [1] and techniques let us do so.

0003-6935/08/340H31-08 $\$ 15.00 / 0$

(C) 2008 Optical Society of America
A possible approach, if we are interested in obtaining high-accuracy spectral estimations in every pixel of an image, would be to build or use a multispectral imaging camera with more than three filters or sensors [1-3]. These systems are really accurate, but they are usually slow (they need long exposure times due to the use of low-transmittance filters in some cases), they are not very cheap (even though they are cheaper than a spectroradiometer), and they suffer from chromatic aberration in some configurations [4]. They are usually built using a monochrome digital camera attached to a device that automatically permits one to select a filter to be used with each of the images (corresponding to the spectral bands) that will be used later to obtain the multispectral image. This device [1] can be a mechanical rotating wheel holding various filters [5], but we can also use a tunable filter, which can be a liquid crystal tunable filter $[1,3]$ or an acoustic optic tunable filter [6]; this permits one to select a transmittance mode for each image taken 
and avoids the problems of rotating wheels regarding alignment and commuting time between different filters. Other possibilities exist that use linear scanning spectral cameras [7] for example. All these multispectral systems are really useful if we want to obtain spectral estimations with similar quality to that of the measurements obtained from a spectroradiometer, but they suffer from some technical drawbacks and costs that make them disadvantageous compared to a customer CCD camera, for example.

In this work we focus on an important natural phenomenon such as skylight $[2,8]$ because researchers in many scientific fields could benefit from highresolution angular maps of skylight's spectral power distribution across the whole skydome. We show here how to obtain real-time, high-resolution skylight radiance spectral power distribution measurements in every pixel of an image of the whole skydome by using a trichromatic CCD camera instead of a spectroradiometer and applying multispectral techniques. We prefer to use this approach instead of using a multispectral system with a larger number of sensors since previous works [3] have proved that spectral skylight can be accurately estimated with only three sensors. We also give some points on how to use this method for studying any other phenomena related to light and color in the open air.

In Section 2 we describe the placement and complete characteristics of the imaging system used in this work. Since we need to train the system in order to apply multispectral techniques for recovering spectra from camera pictures, in Section $\underline{3}$ we present a novel method proposed to select the best set of training spectra from a larger available set. In Section 4 we show the results of the spectral estimations obtained with the system described in previous sections. Finally, Section 5 gives the conclusions and some hints on future applications of such an imaging system.

\section{Description of the Imaging System}

The imaging system $[9,10]$ that we used to obtain spectral estimations of skylight was basically composed of a digital RGB camera (Model Retiga QImaging Q1300, with a 12 bit CCD sensor of 1280x 1024 pixels) attached to a fish-eye lens that permitted us to obtain images of the whole skydome. This system uses a Peltier system as a thermoelectric regulator that controls the temperature inside the housing, keeping it near $25^{\circ} \mathrm{C}$ constantly. All of this structure was protected from rain and wind by a transparent plastic dome and was placed over a moving structure that permitted the Sun to always appear in the central vertical axis of the image. Direct sunlight in the image was avoided by using a black spherical screener moved automatically to hide the Sun reaching the camera.

We show the system in Figs. $1(\mathrm{a})$ and $1(\mathrm{~b})$ and a typical image from this camera in Fig. 2. The imaging system was placed in Granada (Spain, $37.16^{\circ} \mathrm{N}$, $3.60^{\circ} \mathrm{W}, 670 \mathrm{~m}$ altitude), in the rooftop of the Centro Andaluz de Medio Ambiente (CEAMA). This system

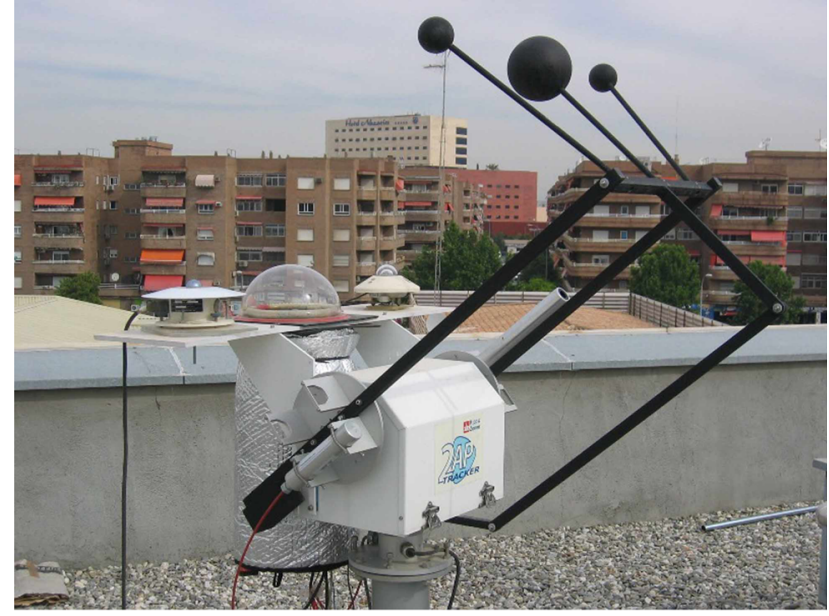

(a)

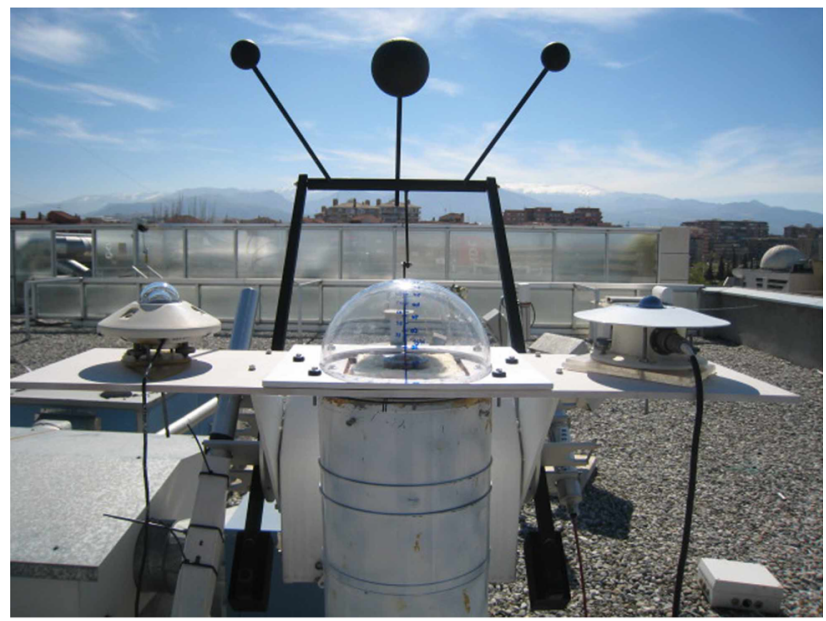

(b)

Fig. 1. (Color online) Complete imaging system used to acquire spectral images of the skydome.

was principally aimed for taking images of the skydome periodically in order to obtain information from clouds and not for multispectral imaging purposes. The acquisition of images could not be interrupted for developing a deep study of the camera from a radiometric point of view. Hence, we were not able to calibrate the camera or change its parameters such as exposure time, gain, or offset. Nevertheless, this is not a big drawback since some spectral estimation algorithms [3] do not need to know the internal specifics of the camera and are robust enough to provide good spectral recoveries even in nonoptimum situations, i.e., with unremoved temporal and spatial noise after a complete calibration of the camera [11,12].

The camera was automatically controlled by remote software and took an image like the one shown in Fig. 2 every 5 min. Exposure time was set to $12 \mathrm{~ms}$ since it was proved that no overexposure is reached in any pixel of the image with this setting. Nevertheless, in some cases some pixels reach and exceed the suggested limit of the $90 \%$ of the maximum achievable 


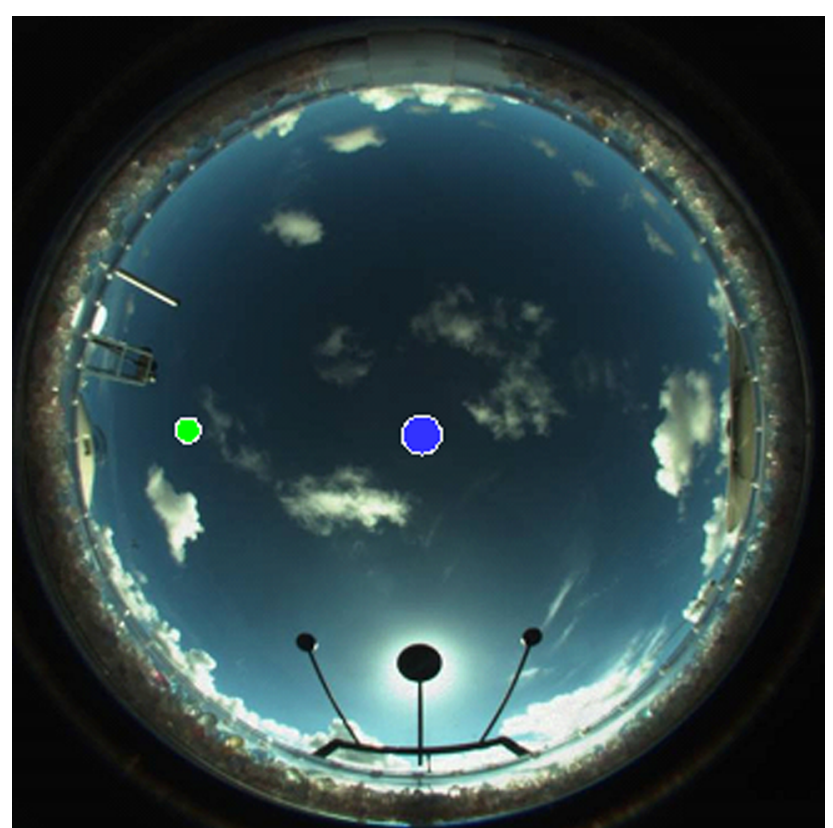

Fig. 2. (Color online) Typical image captured by the CCD RGB camera attached to the fish-eye lens. Note that direct sunlight is avoided by the black sphere. The field of view of the PR650 spectroradiometer corresponds to a circle placed in its corresponding location in the image, with different radius depending on the elevation of the measurement (left, 3 pixels at elevation of $45^{\circ}$; center, 4 pixels at zenith).

value to maintain linearity in CCDs [11,12]. Automatic white balance was turned off. The gain was set to the minimum value for this camera, which is 0.5 , and the offset to 83 . We might remember that 1024 is the maximum recordable value for 12 bit CCD cameras. These gain and offset values seemed optimum from direct visual judgment of the resulting images. We also used a SpectraScan PR650 telespectroradiometer with a field of view equal to $1^{\circ}$ to acquire a spectral measurement of a point in the sky simultaneously with every image from the camera taken. By doing this, we have a complete set of 902 spectral skylight measurements and their corresponding RGB images that are used as a dataset for this study. These measurements were taken in Granada (Spain) at many different points on the skydome and different times of the day, seasons of the year, and weather conditions. In Table $\underline{1}$ we show when these measurements and images were taken, the point of the sky sampled, and the corresponding weather conditions.

Since the fish-eye lens provides a distorted image of the skydome, we must check the correspondence between the area of the sky sampled with the PR650 spectroradiometer (with a field of view of $1^{\circ}$ ) and the exact corresponding group of pixels of the image. We found that the field of view of the PR650 corresponds to a circle of radius equal to 4 pixels when the elevation of the measurement was $90^{\circ}$ (at the zenith), 3.5 pixels when the elevation was $60^{\circ}$, and 3 pixels for elevations of $45^{\circ}$. Two of these cases are shown in Fig. $\underline{2}$ also.

\section{Linear Pseudoinverse Spectral Estimation Method and Training of the System}

\section{A. Training the Linear Pseudoinverse Method}

As we showed in Table 1, we took 902 images of the sky with the CCD camera and their corresponding spectral measurements with the PR650 spectroradiometer in many different situations (see Table 1). Some of these measurements have to be used to train the system (i.e., to build a training set), which means that this new smaller set is used to establish a statistical relationship between the sensors' responses of the camera and the skylight spectra that produced those responses. This relation will be used later to estimate an unknown spectrum, which is not measured with a spectroradiometer simultaneously, from its sensors' responses registered with the camera. If we used the complete set to train and test the system, the results would not be indicative of the behavior of the system in future real situations. Various spectral recovery methods exist that perform this training phase in different ways. In a previous study [3] we compared the speed, accuracy, and robustness of various algorithms, and we showed that the linear pseudoinverse method was preferred due to its simplicity and good results and because it does not need to know the spectral responsivities of the camera's sensors, which in fact are unknown for us in this imaging system we are dealing with (the interested reader can review Refs. [1,11,13] about estimation of sensors' responsivities). The linear pseudoinverse method [3] tries to build a matrix that establishes

Table 1. Measurements Developed with the RGB Camera and the PR650 Spectroradiometer in Granada ${ }^{a}$

\begin{tabular}{|c|c|c|c|c|c|}
\hline Dates & $\begin{array}{c}\text { Number of } \\
\text { Measurements }\end{array}$ & $\begin{array}{c}\text { Elevation } \\
\text { Angle }\end{array}$ & Orientation & $\begin{array}{l}\text { Hours Measuring } \\
\text { (GMT) }\end{array}$ & $\begin{array}{c}\text { Weather } \\
\text { Conditions }\end{array}$ \\
\hline 10 July, 2006 & 37 & $90^{\circ}$ & zenith & $8: 35$ to $18: 00$ & Some clouds \\
\hline 30 October,2006 & 69 & $90^{\circ}$ & zenith & $10: 10$ to $17: 35$ & Some clouds \\
\hline 31 October, 2006 & 38 & $90^{\circ}$ & zenith & $9: 20$ to $12: 30$ & Some clouds \\
\hline 30 November, 2006 & 14 & $45^{\circ}$ & North and South & $15: 50$ to $17: 00$ & Clear \\
\hline 04 December, 2006 & 15 & $45^{\circ}$ & North & $15: 45$ to $16: 55$ & Clear \\
\hline 05 December, 2006 & 275 & $45^{\circ}$ & North, South, East, and West & $9: 10$ to $16: 50$ & Some clouds \\
\hline 12 December, 2006 & 454 & $60^{\circ}$ & North, South, East and West & $9: 00$ to $16: 50$ & Some clouds \\
\hline
\end{tabular}

${ }^{a}$ We show, for each day, the initial and final GMT time while measuring, the number of measurements taken, the elevation angles and orientations of the spectroradiometer, and weather conditions. 
a relationship directly between the sensor responses of the training set, $\rho_{t s}$ (represented by a column vector of $k$ rows, one per sensor), and training spectra, $E_{t s}$ (represented by a column vector of $n$ rows, one per wavelength sample):

$$
W=E_{t s} \rho_{t s}^{+},
$$

where the superscript + refers to the Moore-Penrose pseudoinverse [1]. Thus, once the $n \times k$ matrix $W$ is calculated we can say that the system has been trained, and we can obtain the $n$-rowed vector of the spectral estimations, $E_{R}$, using the linear pseudoinverse method in the way shown in Eq. (2):

$$
E_{R}=W \rho,
$$

where $\rho$ is the $k$-rowed vector of the sensors' responses of a new unknown spectral measurement, which is related to the spectral distribution of light, $E$, impinging on them by

$$
\rho=R^{t} E+\sigma .
$$

Matrix $R$ is $n \times k$ and represents the spectral responsivities of the camera sensors (superscript $t$ indicates transpose), while $\sigma$ is the noise, which is always present in electronic devices, represented by a $k$-rowed vector of unrelated components $[2,3]$.

\section{B. Selection of Training Samples}

Some authors $[1,14,15]$ have proposed various methods in order to select the most appropriate samples from a huge dataset to build a smaller training set. Hardeberg [1] recommended an iterative method where a spectrum is added to the training set to minimize the ratio between the first and the last singular values, which is the condition number. Other authors [14] use the spectra whose values of the cameras responses maximize the average distance with the rest of camera responses values for a given number of samples in the training set. There are other alternative methods $[1,15]$ based on the maximum spectral differences between the training spectra using a certain metric (e.g., entropy [16]) or on using principal component analysis (PCA) to select the directions of maximum spectral variation.

In this work we compare the quality of the training sets obtained with some of these methods against a new proposed method based on using our previously introduced [2,3] colorimetric and spectral combined metric $(\mathrm{CSC} \overline{\mathrm{M}})$, which is calculated as

$$
\mathrm{CSCM}=\operatorname{Ln}(1+1000(1-\mathrm{GFC}))+\Delta E_{a b}^{*}+\operatorname{IRE}(\%),
$$

where $\mathrm{Ln}$ is the natural logarithm; GFC stands for the goodness fit coefficient [8], which is a spectral metric with a value of 1 for perfect matches; $\Delta E^{*}{ }_{a b}$ is the CIELAB distance; and IRE (\%) is the percentage of the integrated radiance error [17], a widely used metric in the field of solar radiation that takes into account differences in the total energy across the visible spectrum. The CSCM metric has proved to be a good candidate for evaluating mismatches between spectra, taking into account three different points of view-spectral, colorimetric, and radiometric - simultaneously. Hence, we will also use this metric to compare the quality of the spectral estimations obtained later.

Our new proposed grouping method takes one random spectrum from the global set of measurements and measures its distance in terms of the CSCM metric to the rest of the spectra in the set. The nearest spectrum to the selected one is grouped to it and deleted from the training set. We then randomly choose another initial spectrum and repeat the grouping process, removing one spectrum at each step, until the desired number of spectra remaining in the training set is reached. This algorithm is fast since we do not need to measure all the distances between the spectra because some of them are calculated in previous iterations and can be reused. We also noticed that, although the concrete set of training spectra selected each time that the algorithm runs is different (due to the random selection of the initial spectrum at each iteration), the quality of the training sets (measured by the quality of the spectral reconstructions obtained when using these sets) is almost the same in all the cases, hence proving the stability in terms of spectral quality of our training set selection algorithm.

As we stated before, our aim in this section is to compare the different methods mentioned for obtaining a training set of spectra against the new proposed one. We use each of these methods (minimization of the condition number [1], closeness to the PCA directions [15], maximization of the entropy [16], and our CSCM-based method) to obtain a training set of 40 samples from the complete data set of 902 skylight spectral measurements taken in Granada. A previous work [3] has proved this number to be sufficient to obtain accurate spectral reconstruction with the linear pseudoinverse method. This complete set is then used as a test set to measure the quality, when using the CSCM metric, of the spectral reconstructions obtained from each training set. In Table 2 we show the results concerning this experiment, where it is clearly seen that the best spectral reconstructions are obtained when we use the training set calculated from our grouping method explained before. In Fig. 3 we show the chromaticity coordinates

Table 2. Mean Values \pm Standard Deviations of the CSCM metric when Recovering the Complete Test Set of 902 Skylight Spectral Measurements Using the Linear Pseudoinverse Method with the Training Set of $\mathbf{4 0}$ Samples Obtained with Each of the Methods

\begin{tabular}{cccc}
\hline $\begin{array}{c}\text { Condition } \\
\text { Number } \\
\text { Minimization }\end{array}$ & $\begin{array}{c}\text { Closeness } \\
\text { to PCA } \\
\text { Directions }\end{array}$ & $\begin{array}{c}\text { Entropy } \\
\text { Maximization }\end{array}$ & $\begin{array}{c}\text { Grouping Spectra } \\
\text { Maximizing } \\
\text { CSCM }\end{array}$ \\
\hline $38.20 \pm 32.97$ & $19.13 \pm 20.89$ & $85.83 \pm 95.21$ & $12.22 \pm 16.70$ \\
\hline
\end{tabular}


of the set of 40 skylight spectra selected by using this proposed method and also the coordinates of the complete data set. We can see that the chromatic variety of the training set expands that of the complete set.

\section{Influence of the Training Set Size}

To end this section, we show some results on the influence of the training set size over the spectral recovery quality. It is interesting to check if there is an optimum number of training spectra to be used in order to obtain accurate spectral reconstructions from the information they provide. This optimum number would be the minimum needed to achieve a desired quality in the spectral recoveries. Some authors $[\underline{1}, \underline{3}, 14,15]$ have studied this parameter, leading, as a main result, to a critical size of the training spectra to be used. Hence, if we use a larger number of training spectra, beyond this critical value, there is no improvement in the results. Curiously, results do not get worse when more and more training spectra are added beyond this critical value, but this is something one would not want to do because the speed of the algorithms decreases with an increasing number of training spectra, very much critically for some concrete spectral estimation methods [3]. We will accept this value as the optimum size of the training set, and-obviously-this optimum size depends on the kind of spectra we are studying, on the spectral recovery algorithm [3], and on the specific hardware of the multispectral system. For instance, Hardeberg [1] found that 20 chosen samples from the Munsell atlas of spectral reflectances are enough to recover accurately the 1269 patches of the complete atlas with a multispectral system based on a CCD monochrome camera and a liquid crystal tunable filter. In previous works [3] we found that this optimum number could be different for different spectral estimation algorithms such as the linear

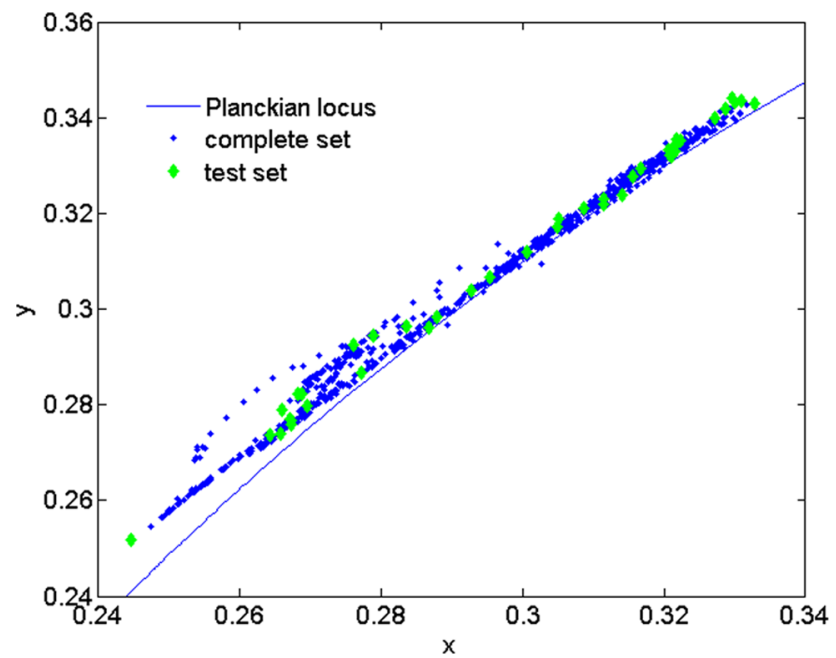

Fig. 3. (Color online) CIE-31 chromaticity diagram showing the $x y$ coordinates of the complete set of 902 skylight measurements taken in Granada (circle) and those $x y$ coordinates of the 40 training samples selected with our grouping method maximazing the CSCM metric (rhombus). The Planckian locus is shown as a line. pseudoinverse or the Shi-Healey [18] method, which typically needed 20 and 150 spectra, respectively, to achieve similar results in high noise situations.

In a new experiment regarding the number of samples of the training set, we use different sizes of this training set, from now on the so-called parameter $m$, obtained by our grouping method explained above. We then recover the complete test set by using again the linear pseudoinverse method with each of the training sets of different sizes. The quality of the spectral estimations is measured once more by means of the CSCM metric, and in Fig. 4 we show the mean values of this metric when the test set of 902 skylight spectral measurements is recovered by using training sets of different sizes in each case. We notice that the improvement in the results for values of $m$ over 100 is not significant, hence we can use this value as the optimum for our system. This optimum value of $m$ may seem high, but we must remind the reader that our set of 902 spectra includes skylight measurements of cloudy and clear days, at many different times of the day and pointing to many different positions of the sky, hence providing a high spectral diversity.

\section{Spectral Estimations Results}

In this section we prove that it is possible to obtain accurate spectral reconstructions from trichromatic RGB CCD camera measurements by using the linear pseudoinverse method and the training set selection method shown in Section 3.A with $m=100$ samples, since this size for the training set has proved to be the optimum (see Fig. 4). The linear pseudoinverse spectral estimation algorithm permits us to obtain spectral curves from the RGB values of the camera, as shown in Eq. [2]. The potential applications of such a result are very interesting, and they are discussed further in this section. We use various metrics to compare the estimated spectral curves from the camera to the measurements obtained simultaneously and at the same point on the skydome from a SpectraScan PR650 telespectroradiometer.

In Table 3 we show the mean and standard deviation values for those metrics composing the CSCM metric as explained in Eq. (4) over the complete test

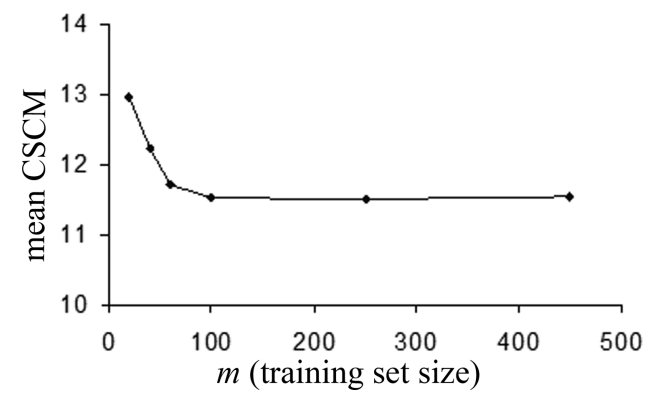

Fig. 4. Mean value of the CSCM metric when recovering the complete test set of 902 skylight measurements taken in Granada by using different training sets of various numbers of samples when using the proposed grouping method. Linear pseudoinverse algorithm is used for the spectral estimations. 
set of 902 skylight spectral measurements. Typical values [2,3] for these metrics, when applied to comparison of very good reconstructions of skylight spectra, are between 0.99 and 0.999 for the GFC, between 1.0 and 3.0 for the CIELAB $\Delta E^{*}{ }_{a b}$ distance and between $2 \%$ and $4 \%$ for the IRE (\%) metric, which gives a range between 4.0 and 9.0 as very good for the CSCM metric. The results obtained with this imaging system estimating skylight spectra are good enough to try to develop other tasks from this stand point. We are trying [19], for instance, to obtain information about climate parameters [20] such as the Angström exponent or the optical depth from the spectral information estimated from the RGB camera images. No existing instrument in this field that we know of is capable of providing measurements of these parameters in every elevation angle at the same time, or even at nighttime, and of course none is as cheap as a RGB CCD digital camera.

We can see that the quality of the spectral estimations obtained with this CCD camera is quite acceptable from spectral and colorimetric points of view. However, the IRE (\%) metric shows not so good results due to a lack of accuracy in the estimation of the total energy impinging on the camera sensors. This effect could be due to the errors introduced by not measuring the variation on the transmisivity of the fish-eye lens with the elevation in the image or by a certain amount of pixel saturation in those images of clear days. Discarding this lack of accuracy in the integrated energy across the visible spectrum, the relative shape of the spectral estimations is quite accurate since GFC values are nearly equal to one. This GFC metric does not take into account different scaling between compared spectra and considers only relative differences in their spectral shapes [21-23].

All these issues are shown in Fig. 5, where two examples of spectral reconstructions obtained with this system are compared to the skylight spectra measured with the spectroradiometer. Figure 5(a) corresponds to the 10th percentile of the CSCM metric (with a value of $\mathrm{CSCM}=2.15$, corresponding to a measurement taken with partially cloudy sky), while Fig. 5(b) corresponds to the 90th percentile of the same metric (with a value of CSCM $=25.56$, corresponding to a clear day and a zenith measurement; the CCD was probably saturated in this image).

We can prove that the quality of the spectral estimations is effectively improved when discarding the differences in total integrated energy by normalizing the spectra (dividing each spectra by its $L^{2}$ norm) before comparing them. By doing this, we obtain the

Table 3. Mean \pm Standard Deviation Values of Various Metrics when Recovering the Complete Test Set of 902 Skylight Spectral Measurements Taken in Granada from the RGB Responses of the CCD Camera ( $m=100$ Training Spectra are Used)

\begin{tabular}{cccc}
\hline GFC & CIELAB $\Delta E^{*}{ }_{a b}$ & IRE (\%) & CSCM \\
\hline $0.9985 \pm 0.0032$ & $0.99 \pm 1.36$ & $9.42 \pm 12.72$ & $11.44 \pm 14.16$ \\
\hline
\end{tabular}

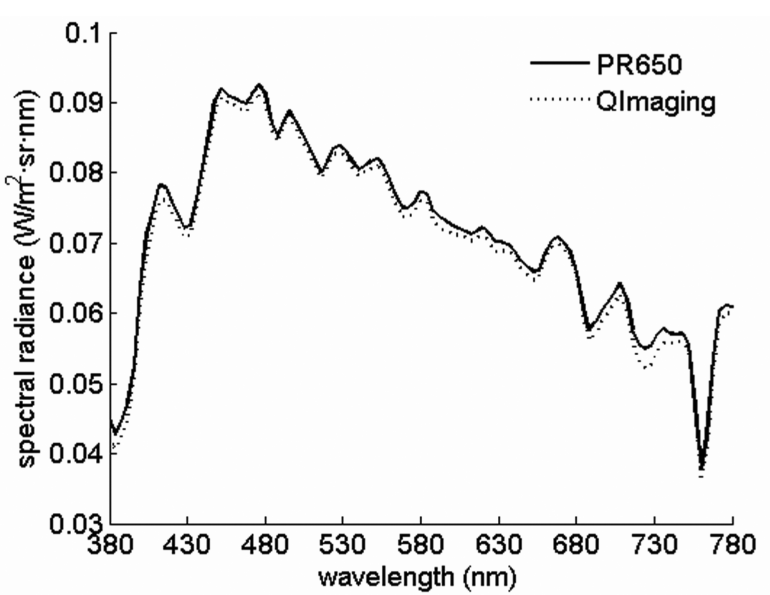

(a)

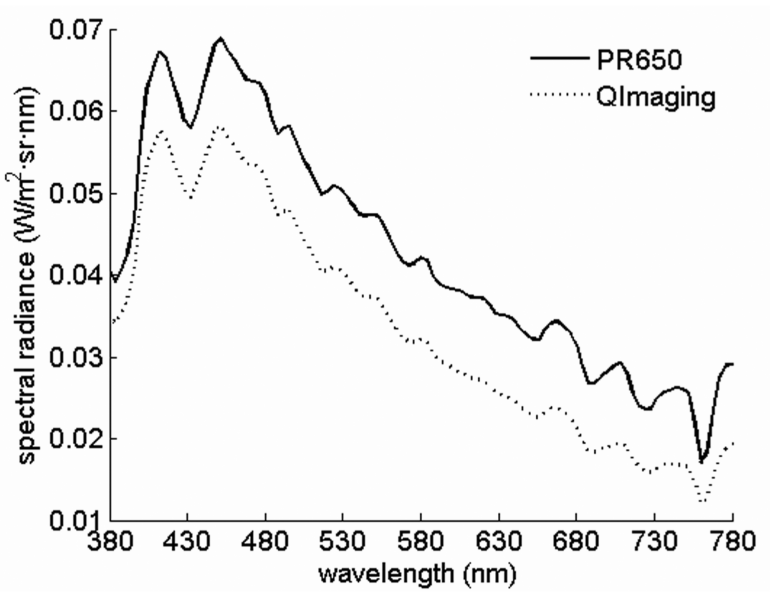

(b)

Fig. 5. (a) 10 th percentile $(\mathrm{CSCM}=2.15)$ and (b) 90 th percentile $(\mathrm{CSCM}=25.56)$ of the CSCM metric over the complete test set of 902 skylight spectral measurements taken in Granada when recovered with the RGB CCD QImaging camera and the linear pseudoinverse method (dotted line) trained with $m=100$ spectra. The spectroradiometric measurement with the PR650 is shown by the solid line.

results shown in Table $\underline{4}$ and Fig. $\underline{6}$, whose CSCM values are quite good considering they were taken from a trichromatic camera measurements. Hence, if we only need to deal with relative spectra and we are not interested in absolute radiometric measurements with this system, we can normalize the results and obtain much better accuracy. This could be done, for instance, if we were interested in performing cloud detection from spectral measurements, since different clouds give different kinds of

Table 4. Mean \pm Standard Deviation Values of Various metrics when Recovering the Complete Test Set of 902 Skylight Spectral Measurements Taken in Granada from the RGB responses of the CCD Camera After Normalization of the Spectral Curves $(m=100$ Training Spectra were Used)

\begin{tabular}{cccc}
\hline GFC & CIELAB $\Delta E^{*}{ }_{a b}$ & IRE (\%) & CSCM \\
\hline $0.9985 \pm 0.0032$ & $1.16 \pm 0.99$ & $0.70 \pm 1.04$ & $2.506 \pm 2.462$ \\
\hline
\end{tabular}




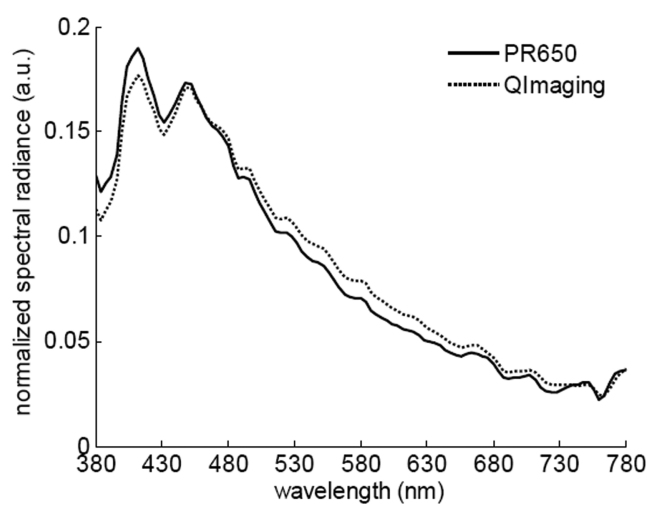

Fig. 6. 90th percentile of the CSCM metric $(\mathrm{CSCM}=5.69)$ over the complete test set of 902 skylight spectral measurements after normalization. The spectroradiometric measurement with the PR650 is shown by the solid line, while the spectral estimation with the Qimaging CCD camera and the linear pseudoinverse method trained with $m=100$ spectra is shown by the dotted line.

spectra, as we can see from Figs. 5(a) (corresponding to a point of the sky with clouds) and 5(b) (corresponding to clear sky zenith).

\section{Conclusions}

In this work we have shown that a trichromatic RGB CCD digital camera can be used to obtain accurate spectral estimations of skylight in a wide variety of situations regarding time of the day, season of the year, weather conditions, and elevation in the image. This kind of imaging system is capable of estimating a spectrum in every pixel of the entire skydome in real time. This spectral information could be used later to perform different calculus to obtain information about climate parameters (such as the Angström exponent or the optical depth [20]), cloud detection and identification, or information regarding different phenomena related to atmospheric optics studies (halos, rainbows, glories, etc.) or natural illuminants.

We have shown that the linear pseudoinverse method [3] is very robust to noise and very appropriate for noncalibrated $[11,12]$ imaging systems where spectral responsivities of the sensors are unknown [3]. It is also a fast method that permits us to obtain one spectrum per pixel in a $1280 \times 1024$ image in real time. We have also proposed a new method for training samples selection, based on grouping similar spectra according to their distances measured by our proposed CSCM metric [2]. This new method for selecting a good training set of spectra performs better than other previously proposed methods. We have shown an experiment where the influence of the training set size has been studied.

Finally, we proved that some tasks regarding spectral information could take advantage of the normalized spectra, which are estimated with higher accuracy with this imaging system. Hence, if no absolute radiometric information is needed, one could obtain really good normalized spectra from the responses of this CCD camera.

\section{References}

1. J. Y. Hardeberg, "Acquisition and reproduction of color images: colorimetric and multispectral approaches," (Dissertation.com, 2001). (Revised second edition of Ph.D. dissertation, Ecole Nationale Supérieure des Télécommunications, 1999), pp. 121-174.

2. M. A. López-Álvarez, J. Hernández-Andrés, J. Romero, and R. L. Lee Jr. , "Designing a practical system for spectral imaging of skylight," Appl. Opt. 44, 5688-5695 (2005).

3. M. A. López-Álvarez, J. Hernández-Andrés, E. M. Valero, and J. Romero, "Selecting algorithms, sensors and linear bases for optimum spectral recovery of skylight," J. Opt. Soc. Am. A 24, 942-956 (2007).

4. S. M. C. Nascimento, F. P. Ferreira, and D. H. Foster, "Statistics of spatial cone excitation ratios in natural scenes," J. Opt. Soc. Am. A 19, 1484-1490 (2002).

5. M. Vilaseca, J. Pujol, M. Arjona, and M. de Lasarte, "Multispectral system for reflectance reconstruction in the near infrared region," Appl. Opt. 45, 4241-4253 (2006).

6. http://micro.magnet.fsu.edu/primer/java/filters/aotf/index .html.

7. J. Antikainen, M. Hauta-Kasari, J. Parkkinen, and T. Jaaskelainen, "Using two line scanning based spectral cameras simultaneously in one measurement process to create a wider spectral area from the measured target," in Proceedings of the IEEE International Workshop on Imaging, Systems and Techniques-IST 2007 (IEEE, 2007), pp. 1-5.

8. J. Hernández-Andrés, J. Romero, and R. L. Lee, Jr., "Colorimetric and spectroradiometric characteristics of narrow-field of view clear skylight in Granada, Spain,” J. Opt. Soc. Am. A 18, 412-420 (2001).

9. A. Cazorla, F. J. Olmo, and L. Alados-Arboledas, "Development of a sky imager for cloud cover assessment," J. Opt. Soc. Am. A 25, 29-39 (2008).

10. A. Cazorla, F. J. Olmo, and L. Alados-Arboledas, "Using a sky imager for aerosol characterization," Atms. Environ. 42, 27392745 (2008).

11. A. Ferrero, J. Campos, and A. Pons, "Low-uncertainty absolute radiometric calibration of a CCD," Metrologia 43, S17-S21 (2006).

12. M. de Lasarte, J. Pujol, M. Arjona, and M. Vilaseca, "Optimized algorithm for the spatial nonuniformity correction of an imaging system based on a charged-coupled device color camera," Appl. Opt. 46, 167-174 (2007).

13. A. Alsam and R. Lenz, "Calibrating color cameras using metameric blacks," J. Opt. Soc. Am. A 24, 11-17 (2007).

14. R. Schettini, G. Y. Novati, and P. Pellegri, "Training set and filters selection for the efficient use of multispectral acquisition systems," in Proceedings of the 2nd European Conference on Colour Graphics, Image and Vision (IS\&T, 2002), pp. $422-426$.

15. M. Mohammadi, M. Nezamabadi, R. S. Berns, and L. A. Taplin, "Spectral imaging target development based on hierarchical cluster analysis", in Proceedings of 12th Color Imaging Conference: Color Science and Engineering, Systems, Technologies and Applications (IS\&T, 2004), pp. 59-64, www.art-si.org.

16. B. P. Lathi, Modern Digital and Analog Communication Systems, 2nd ed. (Oxford University Press, 1989), pp. 132-212.

17. J. J. Michalsky, "Estimation of continuous solar spectral distributions from discrete filter measurements: II. A demonstration of practicability," Sol. Energy 34, 439-445 (1985).

18. M. Shi and G. Healey, "Using reflectance models for color scanner calibration,” J. Opt. Soc. Am. A 19, 645-656 (2002).

19. F. J. Olmo, A. Cazorla, L. Alados-Arboledas, M. A. LópezÁlvarez, J. Hernández-Andrés, and J. Romero, "Proposal of a procedure for the retrieval of the optical depth by an AllSky CCD camera," submitted to Appl. Opt. 
20. R. M. Goody and Y. L. Yung, Atmospheric Radiation, Theoretical Basis, 2nd ed. (Oxford University Press, 1995), Chap. 5.

21. F. H. Imai, M. R. Rosen, and R. S. Berns, "Comparative study of metrics for spectral match quality," in Proceedings of the 1st European Conference on Colour in Graphics, Image and Vision (Society for Imaging Science and Technology, 2002), pp. 492-496.

22. J. A. S. Viggiano, "Metrics for evaluating spectral matches: a quantitative comparison," in Proceedings of the 2nd European
Conference on Colour in Graphics, Imaging and Vision (Society for Imaging Science and Technology, 2004), pp. 286-291.

23. M. A. López-Álvarez, J. Hernández-Andrés, E. M. Valero, and J. L. Nieves, "Colorimetric and spectral combined metric for the optimization of multispectral systems," in Proceedings of the 10th Congress of the International Colour Association (AIC'05), J. Hernández-Andrés and J. L. Nieves, eds. (Association Internationale de la Couleur, 2005), pp. 1685-1688. 